\title{
Are we on track for education and training of future gastroenterologists?
}

\author{
C Mel Wilcox MD ${ }^{1}$, Paul C Adams MD, Editor-in-Chief ${ }^{2}$
}

$D^{\prime}$ C Mel Wilcox is a professor of medicine at the University of Alabama (Birmingham, Alabama, USA), and Director of the Division of Gastroenterology and Hepatology. He is also the Editor-in-Chief of Clinical Gastroenterology and Hepatology.

PA: When we discuss the issues of educating future gastroenterologists, we can look at quantity and quality. What is the ideal number of gastroenterologists?

CMW: One can loosely extrapolate based on the number of practitioners in large metropolitan areas, which averages approximately three to four per 100,000 in the United States (US) but can be much higher. In rural areas, where endoscopic services may be needed, surgeons and even family practitioners in the US are performing endoscopy, but there is real concern regarding the quality of the endoscopic procedures performed by those who have not undergone rigorous endoscopic training. Even if procedures are covered adequately, this still leaves a shortage of gastroenterologists for evaluation and management of gastrointestinal (GI) and liver disorders. We are graduating approximately 400 gastroenterologists from US training programs yearly. This number may be too small if new technologies emerge or unforeseen demands become apparent. Conversely, a momentous discovery that would limit the need for screening colonoscopy could radically alter the number of gastroenterologists required.

PA: Many of our current gastroenterology trainees measure their worth by their endoscopy experience and skills. Is this the correct focus?

CMW: With the increasing need for screening colonoscopy and the disparate reimbursement between procedures, evaluation and management services, endoscopy is the current focus of many practitioners. We all know it is 'easier' to perform a procedure than see someone in the clinic. Indeed, some physicians have their nurse practitioners see patients while they perform procedures, obviously, with the intent of financial gain. This is a bad idea and not good for our patients. Trainees have always believed that high-technology endoscopy or completing some 'procedure' somehow made one special or a 'superstar' - perhaps this is some type of bravado. I believe that being an outstanding diagnostician with compassion is a better goal than becoming a 'technician', although I am an endoscopist. As I have said before, one can teach a monkey to perform endoscopy but such an animal is unlikely to be an empathetic, perceptive physician.

PA: We project the needs of the future based on a large increase in demand for screening colonoscopy. Could this 'bubble' burst down the road?
CMW: A revolutionary diagnostic tool or genetic test abrogating the need for screening colonoscopy would turn the subspecialty on its head overnight. We recognize that such discoveries do not happen quickly - usually there is some warning. Nevertheless, with the breathtaking speed of science, one still wonders. If a genetic test obtained by routine blood sample could identify individuals at highest risk for colorectal neoplasia, then we could focus our resources on such patients. Such a notion has been discussed for some time but is not yet on the horizon. Conversely, if there was some effective, safe pill available that would decrease colorectal neoplasia in those who had a polyp or prevent polyps in high-risk patients, it would also severely limit the need for endoscopic procedures. Whether yet to be developed radiological or blood tests would further reduce the need for endoscopic services is unclear. One may imagine that something similar to capsule endoscopy, which could be performed by internists, would decrease the need for endoscopy epatology performed by gastroenterologists. Science is not standing still.

PA: An American Gastroenterological Association futures initiative suggested that there would be less need for endoscopists in the future and that gastroenterology would become more involved with nutraceuticals.

CMW: This has been stated for some time. As mentioned, there would need to be some reason screening colonoscopy is not needed, especially in the worldwide aging population. It is my opinion that individuals entering the gastroenterology specialty have no desire to discuss nutrition but would rather perform procedures. There is a great need for hepatologists - but why has there not been a coincidental rise in the number of physicians migrating toward this exciting and gratifying subspecialty? This tells me that a focus on nutrition or any other primary evaluation and management service is not going to happen. I do not foresee gastroenterologists increasing their practice load to see obese patients and provide nutritional counselling. If there was some device by which gastroenterologists could get involved with obesity, I think that would be the entry point.

PA: It had been the tradition in large American medical centres for gastroenterology trainees to spend several years in basic science research. Has this changed over time?

CMW: The number of trainees entering a basic science academic career has fallen dramatically. The difficulty in obtaining grant funding may be one reason, but I do not believe it totally explains the plunge. In our centre and many others, the number seeking

\footnotetext{
${ }^{1}$ University of Alabama, Birmingham, Alabama, USA; ${ }^{2}$ University Hospital, London, Ontario

Correspondence: Dr C Mel Wilcox, Department of Medicine, Division of Gastroenterology and Hepatology, University of Alabama,

633 Zeigler Research Building, 730 South 19th Street, Birmingham, Alabama 35294-0007, USA. Telephone 205-975-4958,

fax 205-934-1546, e-mail melw@uab.edu
}

Received and accepted for publication July 20, 2009 
such a track is diminishingly small. This void is being filled by PhDs in other science laboratories. The best chance we have to continue to train MD physicianscientists is to nurture students sooner in medical school or even sooner. Another complementary alternative is to place additional emphasis, as we have in our program, on training clinical academic researchers. There has been a corresponding shift in career choices from the physician-scientist track to the clinician educator and clinical investigator. Academic medical centres have finally realized that a full complement of physicians is required to strike a balance and fulfill the broad goals of research, teaching and clinical care. Clinician educators are needed to train the clinicians of the future as well as house staff at academic medical centres. Clinician investigators are required to perform clinical studies and translational research. This is an area in which we have real opportunities to add physicians to the rank of academicians.

PA: How has the training of clinical researchers changed?

CMW: When I first embarked on an academic clinical investigative path, clinical research was principally a hobby. There were very few courses in methodology. Over the past two decades, however, there has been a growth in the use of a Masters of Public Health-type degree to provide the appropriate training. There should be no surprise that one must obtain adequate training to become a successful clinical investigator, similar to the requirements in the physician-scientist pathway. Indeed, there are more similarities than differences between these two seemingly diverse tracks. The foundation of both is adequate training, opportunities, ideas and mentorship.

PA: If most trainees are headed to clinical endoscopy practice, where does that leave the academic centre?

CMW: Unless significant changes are made, the clinician in the academic medical centre may go the way of the dinosaur. Fortunately, I believe such a situation has been identified at many institutions such that these physicians are now on specific educational or clinical research tracks, and are offered promotion as well as more competitive salaries. In addition, such physicians are gaining respect in an academic setting given that they are greatly needed for patient care and teaching. If not for such physicians, who would take care of the increasing numbers of such patients at academic medical centres in both the inpatient and outpatient settings? Academic clinical practice has may positive attributes. First, it is a privilege to train the physicians of the future and is a huge responsibility. Second, the opportunity to be involved with and surrounded by very bright people who are providing the answers for the future is also exciting. All of us want to stay current and this is an ideal way to do so. Third, one can practice good clinical medicine with exceptionally talented colleagues in our discipline as well as our radiological and surgical colleagues in such centres, as well as provide teaching and mentorship. Finally, one might submit that the lifestyle could be somewhat better and could be a real attribute for the new generation (ie, 'Generation Y'), who may place a greater emphasis on time outside of the work arena rather than salary alone.

PA: Are there any major changes forthcoming regarding training?

CMW: There has been some discussion of extending liver training to two years. I would be interested in your comments, given that you are a hepatologist. As a nonhepatologist, I believe this is a bad idea given the urgent need for more hepatologists, which this requirement would work against. I believe many have now recognized that extending gastroenterology training to three years for someone who just wants to do clinical practice is, in fact, too long. As you know, there is a sixmonth block of research incorporated into the three-year fellowship but, quite frankly, for the trainee headed to clinical practice, the time spent is usually not very productive and does not, I believe, serve a great purpose. There has been some growing consensus to perhaps have those in the third year acquire training in an area of one subspecialty focus. For example, one could train in endoscopic retrograde cholangiopancreatography, endoscopic ultrasound or even hepatology in the third year of training. This could shorten training by one year, which I see as a good thing. There has been intense fascination with natural orifice transluminal endoscopic surgery, but I think it is unrealistic to believe gastroenterologists will be the primary physician here. It is improbable to think that a gastrointestinal endoscopist will be performing any true intraabdominal surgery without ever having appropriate surgical training. 


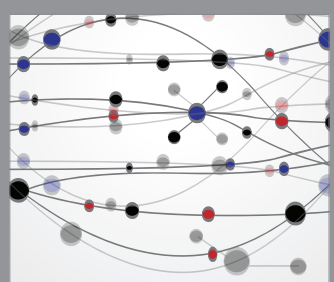

The Scientific World Journal
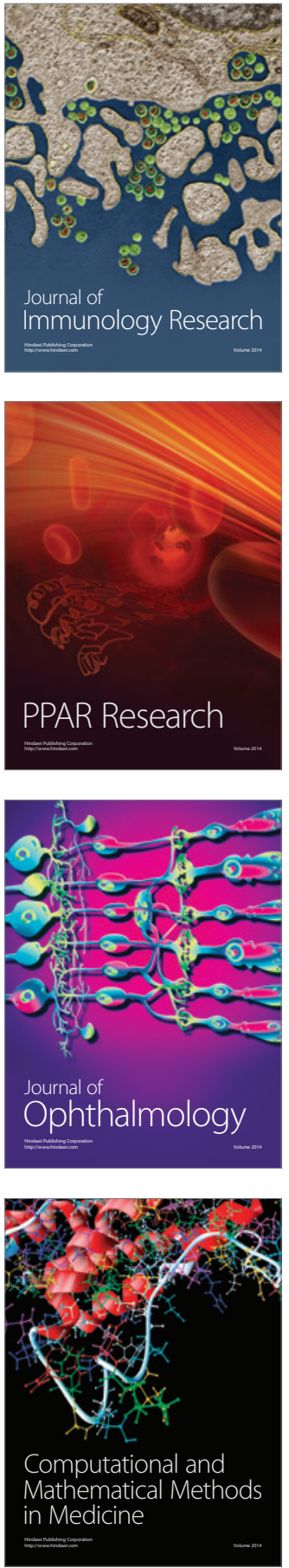

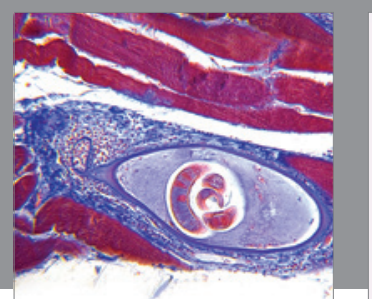

Gastroenterology Research and Practice



\section{Hindawi}

Submit your manuscripts at

http://www.hindawi.com

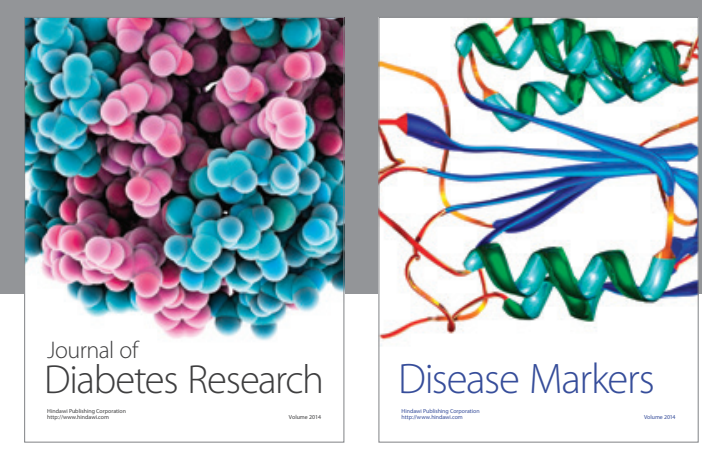

Disease Markers
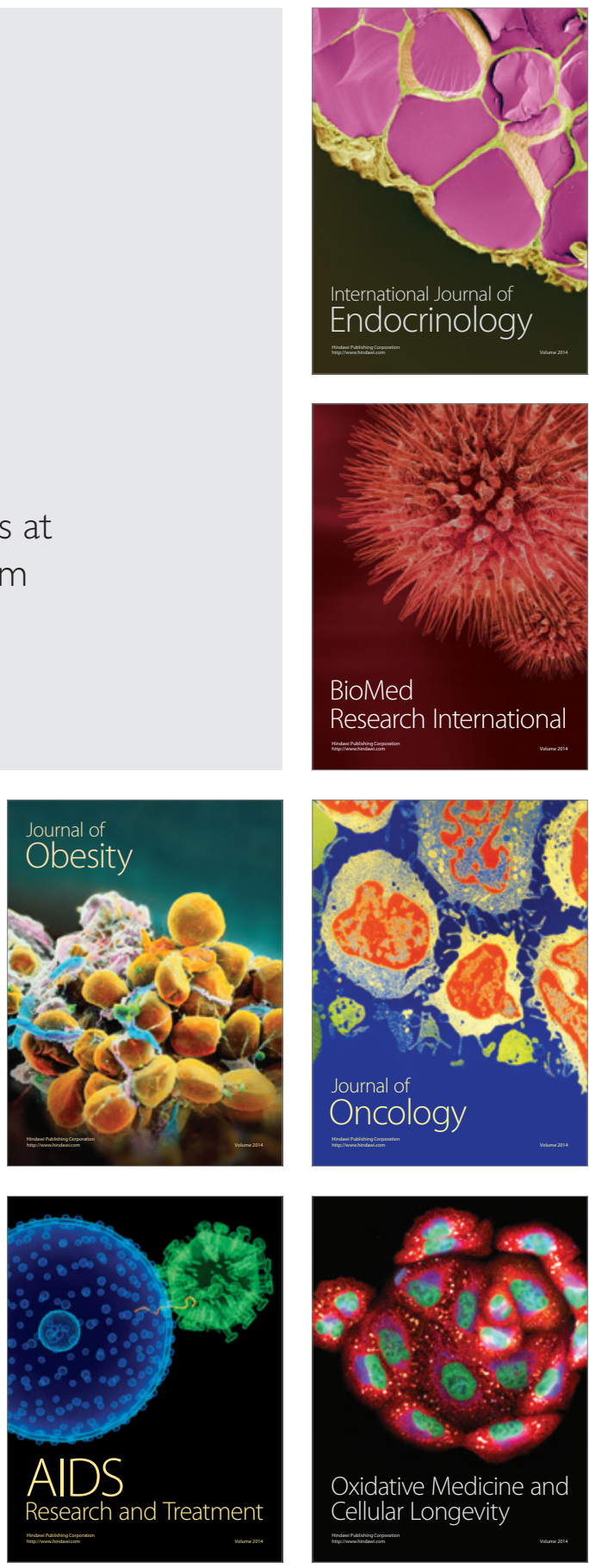